\title{
BMJ Open Study protocol for You.Mind!: boosting first-line mental health care for YOUngsters suffering from chronic conditions with mindfulness: a randomised staggered within- subjects design
}

Merle Kock (D) , ${ }^{1,2}$ Eline Van Hoecke, ${ }^{3}$ Filip Raes, ${ }^{1,2}$ Katleen Van der Gucht ${ }^{1,2}$

To cite: Kock M, Van Hoecke E, Raes F, et al. Study protocol for You.Mind!: boosting first-line mental health care for YoUngsters suffering from chronic conditions with mindfulness: a randomised staggered withinsubjects design. BMJ Open 2021;11:e042648. doi:10.1136/ bmjopen-2020-042648

- Prepublication history for this paper is available online To view these files, please visit the journal online (http://dx.doi. org/10.1136/bmjopen-2020042648).

Received 10 July 2020 Revised 08 March 2021 Accepted 22 March 2021

Check for updates

(C) Author(s) (or their employer(s)) 2021. Re-use permitted under CC BY-NC. No commercial re-use. See rights and permissions. Published by BMJ.

${ }^{1}$ Leuven Mindfulness Centre, KU Leuven, Leuven, Belgium ${ }^{2}$ Centre for the Psychology of Learning and Experimental Psychopathology, KU Leuven Faculty of Psychology and Educational Sciences, Leuven, Belgium

${ }^{3}$ Department of Internal Medicine and Pediatrics, University Hospital Ghent, Gent, Belgium

Correspondence to

Merle Kock;

merle.kock@kuleuven.be

\section{ABSTRACT}

Introduction Adolescents with chronic conditions often experience high levels of stress, anxiety and depression, and reduced quality of life. Mindfulness-based interventions (MBIs) have been found to improve emotional distress in clinical and non-clinical populations and are a promising technique to support adolescents with chronic conditions in managing their symptoms and ultimately enhance their quality of life.

Methods and analysis To test the effects of an MBI on emotional distress and quality of life and delineate the underlying mechanisms, the You.Mind! study uses a randomised staggered within-subjects design. Thirty adolescents with a chronic condition will be randomised to a baseline phase of 14-28 days followed by an MBI, consisting of four online group sessions and online support spread over 8 weeks. Outcomes will be assessed by short, repeated measurements throughout the baseline, intervention and follow-up phases and by standardised questionnaires and experience sampling measures before randomisation, at postintervention and at 3-months followup. Analysis will be based on general linear modelling and multilevel mixed-effects modelling.

Ethics and dissemination Ethical approval was obtained from the Ethics Committee Research UZ/KU Leuven and the Ethics Committee of Ghent University Hospital and Ghent University (S63485). Results will be disseminated through presentations at public lectures, scientific institutions and meetings, and through publication in peerreviewed journals.

Trial registration number NCT04359563.

\section{BACKGROUND}

Adolescents with chronic conditions often experience high levels of stress, which increases risk for anxiety, depression and emotional distress. ${ }^{1-3}$ Additionally, chronic illness and its treatment often limit adolescents' functional ability and reduce their quality of life. ${ }^{4-6}$ This highlights the importance to support adolescents with chronic

\section{Strengths and limitations of this study}

- The You.Mind! study uses a randomised staggered within-subjects design to investigate the effect of a mindfulness-based intervention (MBI) on emotional distress and quality of life.

- Our design allows us to control for the influence of potential time-related confounding variables and the number of baseline measurements, providing stronger evidence that the observed effects are due to the MBI.

- You.Mind! is the first study to investigate the effects of an $\mathrm{MBI}$ in a heterogeneous group of adolescents with chronic conditions.

- Our longitudinal design will enable us to examine the underlying mechanisms of the $\mathrm{MBI}$, but analyses will only be exploratory because of the limited sample size.

- Due to the small sample size, generalisability may be limited.

conditions in managing their symptoms to ultimately enhance their quality of life.

Mindfulness-based interventions (MBIs) have been shown to reduce stress, depression and anxiety and to improve well-being in clinical and non-clinical populations..$^{7-12}$ The concept of mindfulness is described as the compassionate, non-judgmental moment-to-moment awareness of one's experiences. ${ }^{13}$ Mindfulness skills are commonly taught using structured interventions such as mindfulness-based stress reduction (MBSR) ${ }^{14}$ or mindfulness-based cognitive therapy (MBCT). ${ }^{15}$ Within this structured curriculum, participants develop skills that enable them to become non-judgmentally aware of thoughts, feelings and sensations, and to 
replace automatic, habitual and judgmental reactions with conscious, skilful and compassionate responses.

Recent reviews suggest that MBIs are a promising intervention to support adolescents with chronic conditions. ${ }^{1617}$ Overall, the authors conclude that MBIs are acceptable and feasible, and that there is some evidence suggesting that MBIs can improve emotional distress among adolescents with chronic conditions. However, considering the small sample sizes, heterogeneity of measures, and lack of randomisation and control groups in previous studies, more robust research is needed to replicate these findings. Moreover, the working mechanisms underlying MBIs are currently unclear. In a previous study, we investigated the impact of an 8-week MBI in adolescent cancer survivors and found significant improvements in quality of life and reductions in emotional distress, which were associated with reductions in negative attitudes towards the self and increases in mindfulness skills. ${ }^{18}$ However, the substantive time commitment necessary to complete the MBI was problematic for some participants. As an alternative, a recent study investigated an E-health MBI and found that it reduces depressive symptoms and has a positive impact on well-being, stress, pain control and sleep in adolescents with a chronic illness. ${ }^{19}$ These findings suggest that electronic MBIs delivered to adolescents with various chronic conditions are feasible and acceptable but further research is necessary to investigate the effectiveness of such interventions. ${ }^{17}$

An efficient way to robustly investigate the effects of MBIs on emotional distress is by using a randomised staggered baseline design. This design allows to test causal relations between intervention and outcome variables since changes in outcome variables in the intervention period are being compared with changes in outcome variables during the baseline period for each individual participant. Thus, each participant acts as its own control. The internal validity of this design is further strengthened by randomising participant groups to different intervention starting points and testing whether a similar change pattern occurs across groups after the introduction of the intervention. ${ }^{20}$ Compared with group-based designs, randomised staggered baseline designs require smaller samples but more measurement points to achieve the same power, making them most suitable for populations that are hard to recruit but can be easily measured repeatedly. ${ }^{21}$ Finally, consistent with the trend towards personalised medicine, single-case designs such as a randomised staggered baseline design allow to evaluate interventions in a person-centred manner. According to Hofmann and Hayes, ${ }^{22}$ intervention science is starting to refocus towards an individual's needs and strengths. They argue that this change of focus is key for the future of evidence-based care to enable efficient and effective treatment change. Single-case designs are vital to enable individualised analysis and drive the change towards personalised evidence-based treatment. ${ }^{22}$ With regard to MBIs, single-case designs allow for a robust test of the effects of MBIs in adolescents with chronic conditions including randomisation and a control phase, which were often lacking in prior studies, ${ }^{16}$ while keeping the sample size low. Moreover, such a design and the inclusion of experience sampling methods (ESM) would enable an examination of the trends of the outcome variables over the course of treatment and allow for a more ecologically valid outcome assessment. ${ }^{23}$ This would help to understand the individual change processes of participants across treatment and identify the minimum amount of mindfulness training necessary for benefits to occur. ${ }^{16}$

\section{OBJECTIVES AND TRIAL DESIGN}

The present study aims to investigate the effect of an MBI on emotional distress and quality of life in adolescents with chronic conditions and to evaluate the underlying mechanisms. We will apply a blended care approach consisting of four online group sessions spread over 8 weeks with the use of a mobile application in-between sessions. In this way, difficulties regarding time commitment noted in previous studies will be addressed, and acceptability and practicability of our MBI will be improved. ${ }^{16-18}$ Furthermore, adolescents with various types of chronic conditions will be invited for participation to assess efficacy in a more diverse sample compared with prior research. ${ }^{17}$ The secondary objective is to evaluate the underlying mechanisms of the MBI by replicating and extending our previous findings. The You.Mind! study uses a randomised staggered within-subjects design. Thirty adolescents with a chronic condition will be randomised to a baseline assessment phase of 14-28 days followed by the MBI. Outcomes will be assessed by short repeated measurements throughout the baseline, intervention and follow-up phases, and by standardised questionnaires and experience sampling measures before randomisation, directly post-intervention and at 3-months follow-up. Additionally, interviews will be conducted before and after the MBI to explore participants' experience.

\section{METHODS AND ANALYSIS \\ Participants}

Participants will be recruited by staff from the paediatric unit of the University Hospital Ghent, Belgium. Adolescents meeting the following criteria will be included in the study: (1) 14-19 years of age; (2) suffering from a chronic condition lasting 1 year or more that impairs functional mobility and/or requires ongoing medical care; (3) sufficient proficiency in the Dutch language. Adolescents who suffer from a current or lifetime severe psychiatric disorder or who are currently receiving treatment for a psychiatric disorder will be excluded. Participants will continue to receive their usual care for their respective physical condition including support from a psychologist, if applicable. Data collection will start on 21 July 2020 and is expected to finish until 31 December 2022.

\section{Sample size}

Since there is no consensus on how to conduct a power analysis for the present design and statistical method, the 
power of our multilevel analysis can be approximated by the power of a repeated measures analysis of variance (ANOVA) with one within-subjects factor time. For the effect on quality of life, we conducted a power analysis with three measurement points (pre- vs post- vs follow-up assessment) in a comparison of an A-phase and B-phase (two groups) with $\alpha=0.05$ and $80 \%$ power to detect a medium effect size based on our previous study on the effects of an MBI on cancer survivors. ${ }^{18}$ If we assume a realistic correlation of 0.5 between repeated measurements, 28 participants are necessary to reach sufficient power. For the effect on emotional distress, another power analysis was conducted with 63 measurements (throughout baseline, intervention and follow-up phases) and the other variables as specified above, yielding a required sample size of 6. However, repeated measures ANOVA assumes sphericity, while multilevel modelling does not. Moreover, multilevel modelling takes the sampling hierarchy into account, can analyse missing data and has more statistical power according to simulation studies. ${ }^{24}$ Taken together, this suggests that the proposed study with a sample size of 30 (before attrition) has sufficient power of at least $80 \%$ to detect the hypothesised medium effect on primary outcomes. Given our experience with recruitment of clinical adolescent populations (ie, cancer survivors) and the strong cooperation with the hospital personnel, we are convinced that recruitment of 30 participants will be feasible in the time frame of 2.5 years.

\section{Mindfulness-based intervention}

The MBI will be delivered using a standardised protocol that we developed ${ }^{18}$ on the basis of $\mathrm{MBSR}^{14}$ and $\mathrm{MBCT}^{15}$ manuals with adjustments for adolescent populations. Adjustments were informed by our extensive experience with mindfulness in adolescent populations across different contexts. The essential aim of the MBI is to increase awareness of one's experience in the present moment and to establish an open and accepting attitude towards one's experiences. By practicing this attitude, participants begin to form a detached and non-reactive relationship with their experience. Participants learn to recognise entanglement in one's thoughts and emotions, and understand the own tendency to react in spontaneous, habitual ways. Together, these skills enable participants to deal with problematic thoughts and emotions instead of strengthening them. As the central foundational methodology, mindfulness meditation is extensively trained throughout the MBI. ${ }^{25}$

The blended care programme consists of four 2-hour online group sessions spread over 8 weeks. In-between sessions, participants use a mobile application to assist their home practice. Each session includes guided experiential mindfulness exercises, such as focus on the breath, body scan, breathing space, mindfulness yoga, insight meditation or walking meditation, sharing of personal experiences with the exercises, reflections in small groups, psychoeducation regarding stress, depression and self-care, and a review of home practices. For a more detailed overview of the core elements in each session, see van der Gucht $e t a l^{26}$ The curriculum is available in open source (https://ppw.kuleuven.be/home/ mindfulness/adolescents/). The MBI will be delivered by highly qualified, certified trainers with more than 10 years of experience in mindfulness meditation and extensive experience working with adolescents. Trainers will attend regular intervision and supervision moments to check treatment fidelity and trainer adherence. Groups will include five participants, yielding six groups in total. Participant adherence will be monitored using session attendance rates and usage of homework audio files on the application. Participants can withdraw from the intervention at any time and without being obliged to give any reason.

\section{Outcomes}

Primary outcomes are emotional distress, defined as the level of depression, anxiety and stress measured through ESM (see below), and quality of life measured through self-report questionnaires. Secondary outcomes address our second aim, that is to evaluate mechanisms that drive change in primary outcomes following the MBI; these include anhedonia, dampening, acceptance/suppression of negative emotions, repetitive negative thinking, selfcompassion, all retrieved from experience sampling, as well as acceptance of the chronic condition and mindfulness skills assessed via self-report questionnaires. Outcome variables assessed using ESM will additionally be evaluated using self-report questionnaires as extra validation of ESM assessments. Outcomes will be analysed as change from baseline. Furthermore, adverse events, frequency of home practice and usage of the application will be assessed.

\section{Experience sampling}

ESM is a validated, structured diary technique that assesses participants within their daily environment. This method was chosen because of its high ecological validity and short completion time, allowing for in-the-moment measurements in a prospective manner over a longer time period. ${ }^{23}$ During the main assessments, ESM questions will be asked 10 times/day for 4 consecutive days according to a semistratified interval scheme (waking hours will be divided into 10 equal intervals and in each interval, one beep will be randomly programmed). In-between the main assessments, ESM questions will be asked daily (baseline phase) or every 3 days (intervention and follow-up phases) at 17.00 to monitor changes over time. At each beep, participants will be asked to indicate their current experience of emotional distress (three items), suppression/acceptance of negative emotions (two items), anhedonia (three items), dampening (one item), repetitive negative thinking (one item) and selfcompassion (one item).

\section{Self-report questionnaires}

Quality of life will be assessed using the total score on the adolescent version of the Pediatric Quality of Life 
Inventory (PedsQL 4.0). ${ }^{27}$ The 23-item PedsQL 4.0 Generic Core Scales consist of four subscales: physical, emotional, social and school functioning. Items are rated on a five-point Likert scale and rescaled to a $0-100$ scale, where higher scores indicate better quality of life. The Dutch version used in this study has shown good psychometric properties. ${ }^{28}$

Emotional distress will be measured using the total score of the Depression Anxiety Stress Scales (DASS-21).$^{2930}$ The scale consists of three seven-item subscales that measure symptoms of depression, anxiety and stress over the past week. Items are scored on a four-point Likert scale with higher scores reflecting higher emotional distress. The DASS-21 evidenced good psychometric properties in the Dutch version. ${ }^{29} 31$

The 21-item Leuven Anhedonia Self-report Scale ${ }^{32}$ will be used to assess consummatory, anticipatory and motivational aspects of anhedonia over the past 2 weeks. Items are scored on a five-point Likert scale with higher scores reflecting greater anhedonia.

The dampening subscale of the Reponses to Positive Affect (RPA) ${ }^{33}$ questionnaire will be used to measure dampening responses to positive affect. Its seven items are rated on a four-point Likert scale, where higher scores reflect greater dampening. The Dutch version of the RPA evidenced good psychometric properties. ${ }^{34}$

The Non-Acceptance and Suppression of Negative Emotions Scale (Raes F 2019, unpublished) is a 10-item scale designed to assess the extent of suppression compared with acceptance of negative emotions on a seven-point Likert scale. Higher scores indicate stronger suppression of negative emotions.

The core characteristics subscale of the Perseverative Thinking Questionnaire ${ }^{35}$ will be used to assess the main characteristics of repetitive negative thinking: repetitiveness, intrusiveness and difficulty to disengage. Its nine items are scored on a five-point Likert scale with higher scores indicating greater repetitive negative thinking. The Dutch version evidenced good psychometric properties. ${ }^{36}$

The short form of the Self-Compassion Scale (SCS-SF) ${ }^{37}$ will be used to measure participants' self-compassion. Its 12 items are scored on a six-point Likert scale, where higher scores reflect greater self-compassion. Good psychometric properties have been reported for the Dutch version of the SCS-SF. ${ }^{37}$

The adolescent version of the Comprehensive Inventory of Mindfulness Experiences (CHIME-A) ${ }^{38}$ will be used to assess eight different aspects of mindfulness: awareness of internal experiences, awareness of external experiences, acting with awareness, accepting and nonjudgmental attitude, non-reactive decentering, openness to experiences, awareness of thought's relativity and insightful understanding. Its 24 items are scored on a sixpoint Likert scale with higher scores indicating greater mindfulness skills. The CHIME-A evidenced good psychometric properties. ${ }^{38}$

An adapted version of the Pain Solutions Questionnaire $(\mathrm{PaSol})^{39}$ will be used to measure participants' acceptance of their condition. Its 14 items are rated on a seven-point Likert scale. The PaSol evidenced good psychometric properties. $^{39}$

\section{Harms}

At intake and all main assessment points, participants will be provided with information where to find counselling. All solicited and spontaneously reported adverse events and other unintended effects of the study will be collected, assessed, reported and managed according to good clinical practice guidelines. The Harms and Unpleasant Experiences Survey (Baer 2020, unpublished) will be used to monitor possible adverse events that happened during the intervention period. For openended assessment of difficulties and strengths of the MBI, participants will describe three positive and three negative aspects of their mindfulness practice at follow-up assessment. This will complement previous research that largely failed to actively assess adverse events in psychological interventions. ${ }^{40}$

\section{Procedure}

Potential candidates will be contacted by paediatric psychologists working at the University Hospital Ghent. Interested patients will be contacted by phone and eligibility will be checked (see table 1). Potential participants will receive information and consent forms for themselves and their parents. They will have the opportunity to ask questions and discuss participation with their parents before giving consent. Potential participants will also be invited for an intake interview with the mindfulness trainer. In case of risk for psychopathology, participants will be excluded and referred to professional help.

Once 15 participants have been enrolled, they will be randomised to one of three MBI groups, which start their intervention with 0.5 weeks time lag (see figure 1 ). Within each group, participants will be randomised to a baseline phase of $2,2.5,3,3.5$ or 4 weeks, with 3-4days between starting points of individual participants, using a computer-generated randomisation procedure. The same procedure will be repeated for the second pool of 15 participants. This design allows to robustly test the causal relationship between the MBI and outcome variables in two ways. ${ }^{41}$ First, the extent to which the trend observed in the outcome variables at baseline differs from the trend observed after the introduction of the intervention provides evidence that the observed pre-post changes result from the intervention. Second, randomising first the groups to different intervention starting points and afterwards individual participants to different baseline lengths enables us to introduce a staggered randomised baseline design in the presence of a group intervention. In this way, participants from different groups may start their baseline phase at the same time while having baseline phases of varying lengths, similar to an individual staggered randomised baseline design. Changes in outcome variables in one participant after the start of the intervention coupled with the absence of change in other 
Table 1 Participant timeline throughout the study

\begin{tabular}{|c|c|c|c|c|c|c|c|c|}
\hline \multirow[b]{2}{*}{ Time point } & \multicolumn{8}{|c|}{ Study period } \\
\hline & Enrolment & Allocation & A1 & $\begin{array}{l}\text { Baseline } \\
\text { phase }\end{array}$ & $\begin{array}{l}\text { Intervention } \\
\text { phase }\end{array}$ & A2 & $\begin{array}{l}\text { Follow-up } \\
\text { phase }\end{array}$ & A3 \\
\hline \multicolumn{9}{|l|}{ Enrolment } \\
\hline Eligibility screen & $\mathrm{x}$ & & & & & & & \\
\hline Intake interview & $x$ & & & & & & & \\
\hline Informed consent & $x$ & & & & & & & \\
\hline $\begin{array}{l}\text { Allocation to baseline } \\
\text { phase }\end{array}$ & & $x$ & & & & & & \\
\hline \multicolumn{9}{|l|}{ Intervention } \\
\hline $\begin{array}{l}\text { Mindfulness-based } \\
\text { intervention }\end{array}$ & & & & & $x$ & & & \\
\hline \multicolumn{9}{|l|}{ Assessments } \\
\hline Self-report questionnaires & & & $x$ & & & $x$ & & $\mathrm{X}$ \\
\hline Harm assessment (HUES) & & & & & & $x$ & & \\
\hline $\begin{array}{l}\text { Harm assessment } \\
\text { (positive/negative aspects) }\end{array}$ & & & & & & & & $x$ \\
\hline $\begin{array}{l}\text { Home practice and } \\
\text { application usage }\end{array}$ & & & & & & $x$ & & $x$ \\
\hline $\begin{array}{l}\text { ESM (10 times/day for } \\
4 \text { days) }\end{array}$ & & & $x$ & & & $x$ & & $x$ \\
\hline ESM (daily) & & & & $\mathrm{x}$ & & & & \\
\hline ESM (every 3 days) & & & & & $x$ & & $x$ & \\
\hline Interview & & & & $x$ & & & $x$ & \\
\hline
\end{tabular}

A1, pre-assessment; A2, post-assessment; A3, follow-up assessment; ESM, experience sampling method; HUES, Harms and Unpleasant Experiences Survey.

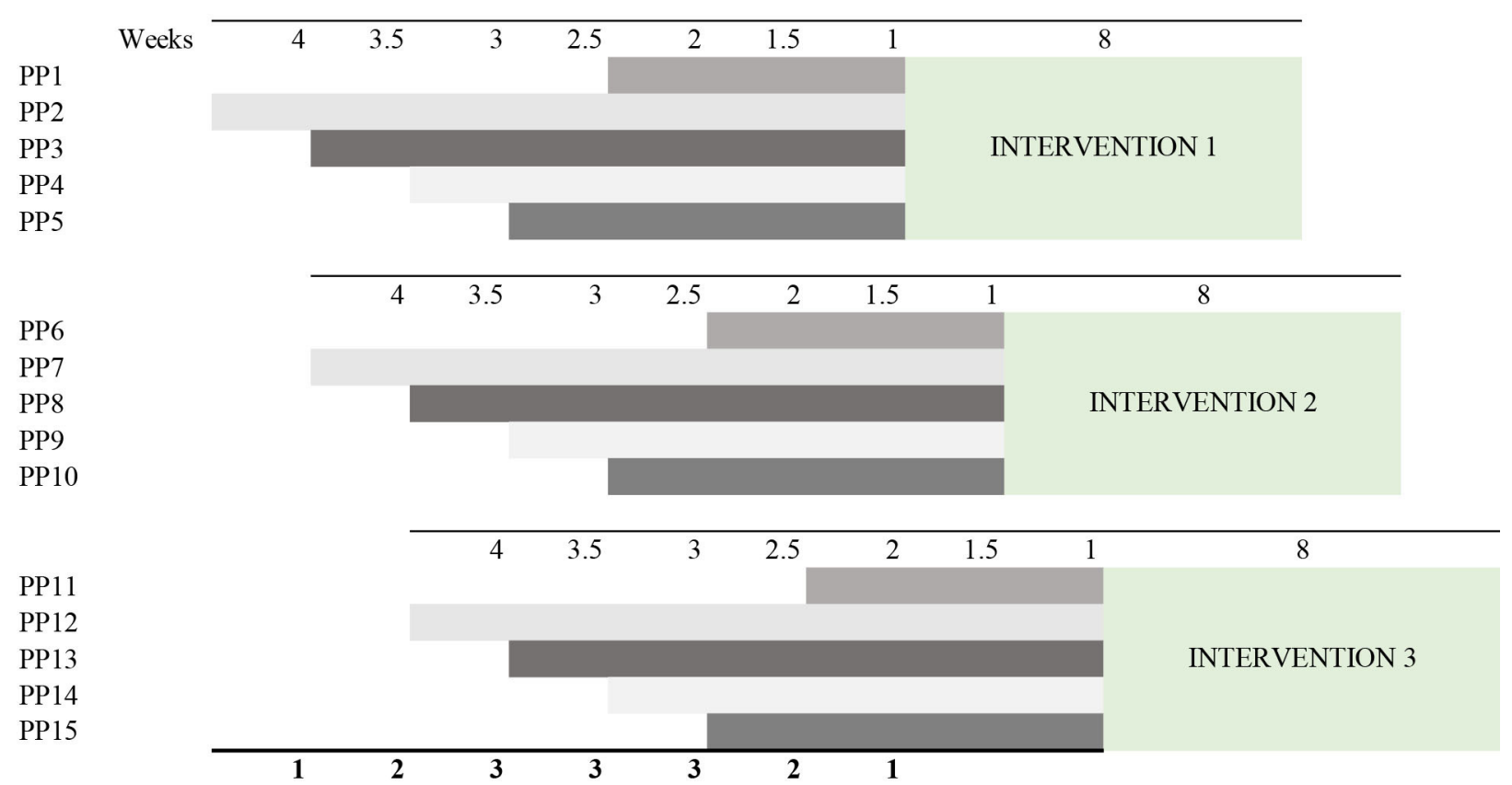

Figure 1 Schematic overview of the baseline and intervention phases for each participant. Participants within each intervention group are randomised to a 2-4 week baseline phase starting at different time points. The mindfulness intervention commences directly after the baseline phase with half a week time lag between intervention groups. Across groups, participants are starting their baseline phase at the same time but begin their intervention at different time points. 
participants after the same number of measurement days who have not received the intervention yet provide strong evidence that changes result from the intervention. Using this design, we control for the influence of potential timerelated confounding variables and the number of baseline measurements.

After the baseline phase, participants will follow the MBI consisting of four group sessions spread over 8 weeks. Outcomes will be assessed in two ways. Extensive assessments will take place before randomisation (preassessment), directly post-intervention (post-assessment) and at 3-months follow-up (follow-up assessment) and include self-report questionnaires and ESM measures 10 times/ day for 4 consecutive days. In between these assessments, during baseline, intervention and follow-up phases, participants will complete ESM items daily (baseline) or every 3 days (intervention and follow-up). Participants will have 24 hours (questionnaires and daily ESM) or 10 min (extensive ESM) to complete assessments. To improve protocol adherence, participants will receive reminders for each of their assessments. For daily assessments, participants will be provided with strategies to remember assessments. Up to 14 days before the beginning and after the end of the intervention, participants will be invited for interview to assess motivation and expectations (first interview) as well as personal experiences and possible benefits of the MBI (second interview). After study completion, participants will receive a voucher of $20 €$ as an incentive. Participants who discontinue the intervention will be invited to share their reasons to drop out and take part in follow-up assessments on a voluntary basis. All participants will have continuous access to the smartphone application after study completion.

\section{Patient and public involvement}

Due to the young age of our target population, there has been no patient and public involvement in this study.

\section{Data management}

After providing informed consent, participants will receive a unique, anonymised participant-ID. As this study includes multiple waves of data collection, a datafile that links the participant-ID to their name will be stored throughout the study in a separate restricted online environment. On completion of the study, this datafile will be deleted. After data collection and before data storage, all outcomes are manually double-checked by research staff. The safety, progress, study integrity and design aspects will be monitored by the research team. Given the known minimal risks of the study and the short duration for each participant, a formal data monitoring committee is not considered necessary.

Types of data that will be collected:

- Restricted and personal information including the datafile linking participants' name to participant ID, month and year of birth, contact information, signed informed consent forms. This information will be stored at a restricted area of the primary researcher's personal drive in an in-house protected server at $\mathrm{KU}$ Leuven, which can only be accessed by the involved researchers.

- Confidential data including age, demographic data, all other data coming from questionnaires, experience sampling data and interviews. This data will be pseudoanonymised and stored in a database in an in-house protected server at KU Leuven. To allow for secure storage, management and sharing, and to avoid loss of data and/or conflicting versions, we will use a shared drive. It allows involved research team members to store and edit files, and to access the information using their employee ID. Collaborators at University Hospital Ghent will not have access to the data. Only researchers directly involved in data analysis will have access to the final pseudoanonymised data set. Pseudoanonymised data will not be transferred unencrypted or by e-mail, but can be shared with regulatory authorities, ethical committees and other parties that collaborate with the research team.

- Special note on data pertaining to the mindfulness mobile application: the application will administer the ESM-questions and register participants' responses. The application will also log usage data, including application starting times, visited audio and videos together with the duration of the interaction and application error logging. All data will be sent to a server together with the pseudonym ID, using encrypted https protocol. The server and all the data will be located at and managed by KU Leuven. The application and the server will use the pseudonym ID to link the data and never contain personal data or the actual identification of the person. The logging stops when data collection stops.

\section{Statistical analysis plan}

To evaluate the effect of the MBI on primary and secondary outcomes, different multilevel model analyses will be conducted. For the ESM phase assessments (in-between main assessments), we will use a piecewise model for intercept/slope discontinuity as proposed by Prinz et $a l^{42}$ For the self-report and ESM main assessments, a piecewise multilevel model based on our previous research will be used. ${ }^{43}$ As an exploratory analysis, we will perform mediation analyses to examine whether any secondary outcome drives the effect of the MBI using a lower level time-lagged-mediation model based on our previous study. ${ }^{43}$ The full maximum likelihood estimator will be used to handle missing data. Number of attended sessions and completed homework assignments will be added as covariates. Results will be controlled for multiple testing using the false discovery rate. ${ }^{44}$

Harm outcomes will be analysed using descriptive statistics. Interviews will be audiotaped, transcribed and coded separately for preintervention and postintervention interviews using Nvivo V.11. On the basis of these codes, a thematic analysis will be conducted. 


\section{ETHICS AND DISSEMINATION}

\section{Ethical considerations}

Ethical approval for this study was obtained at the Ethics Committee Research UZ/KU Leuven (S63485, leading committee) and the Ethics Committee of Ghent University Hospital and Ghent University. They reviewed study design, ethics and all participant-facing documents. The research team will provide an annual progress report and a clinical trial report to the ethics committees within 3 months after completion of the study, including the date of last trial-related procedure performed, final number of participants and events of special interest. The Ethics Committee Research UZ/ KU Leuven will review the study at least annually. Any subsequent modifications to the protocol that may impact trial conduct or affect participant safety will be agreed on by the research team and submitted to both ethics committees before implementation. All amendments will be reported to ClinicalTrials.gov and the editors of the present paper.

\section{Dissemination plan}

Research results will be disseminated through presentations at locally organised public lectures, scientific institutions, patient organisations and conference meetings, and through publication in peer-reviewed journals. For authorship, guidelines of the respective journal will be followed. Results will be disseminated regardless of magnitude or direction of the observed effect. The full pseudoanonymised data set will be shared beginning 3 months and ending 5 years following publication of the results. Access will be granted to interested researchers for any type of analysis if they agree with the confidentiality rules agreed on in this study. To gain access, contact the authors at merle.kock@kuleuven.be. Study protocol, statistical analysis plan and analytical code will be shared via the following link beginning 3 months and ending 5 years following publication of the results: https://clinicaltrials.gov/ct2/show/NCT04359563? term=katleen+van+ der+gucht\&draw=2\&rank=3.

\section{Twitter Merle Kock @KockMerle}

Acknowledgements We would like to thank Patrick Onghena for acting as our statistical consultant and Peter Kuppens for his support during the revision process.

Contributors MK, KVdG and FR were involved in conception and study design. KVdG applied for funding. MK was responsible for drafting the article. EVH will be responsible for recruitment. MK and KVdG will conduct data collection and analysis. All authors were involved in critical revision of the article for important intellectual content, approved the final version of the article and agreed to be accountable for all aspects of the work.

Funding This work is supported by Fondation Huoshen. The support includes financial funding over the whole period of the trial. The sponsor and the funding source were not involved in the design of this study and will not have any role during its execution, analyses, interpretation of the data or decision to submit results. MK is supported by a Red Noses grant (G049019N) from the Research Foundation—Flanders (FWO-Vlaanderen).

Competing interests KVdG and FR are founders and member of the managing committee of the Leuven Mindfulness Centre Fund; FR and KVdG receive payments for workshops and presentations related to mindfulness. The authors declare that they have no other competing interests.
Patient and public involvement Patients and/or the public were not involved in the design, or conduct, or reporting or dissemination plans of this research.

Patient consent for publication Not required.

Provenance and peer review Not commissioned; externally peer reviewed.

Open access This is an open access article distributed in accordance with the Creative Commons Attribution Non Commercial (CC BY-NC 4.0) license, which permits others to distribute, remix, adapt, build upon this work non-commercially, and license their derivative works on different terms, provided the original work is properly cited, appropriate credit is given, any changes made indicated, and the use is non-commercial. See: http://creativecommons.org/licenses/by-nc/4.0/.

ORCID iD

Merle Kock http://orcid.org/0000-0001-9429-6321

\section{REFERENCES}

1 Barlow JH, Ellard DR. The psychosocial well-being of children with chronic disease, their parents and siblings: an overview of the research evidence base. Child Care Health Dev 2006;32:19-31.

2 Delamater AM, Guzman A, Aparicio K. Mental health issues in children and adolescents with chronic illness. IJHRH 2017;10:163-73.

3 Pinquart M, Shen Y. Depressive symptoms in children and adolescents with chronic physical illness: an updated meta-analysis. $J$ Pediatr Psychol 2011;36:375-84.

4 Lavigne JV, Faier-Routman J. Psychological adjustment to pediatric physical disorders: a meta-analytic review. J Pediatr Psychol 1992;17:133-57.

5 Santos T, de Matos MG, Simões C, et al. (Health-related) quality of life and psychosocial factors in adolescents with chronic disease: a systematic literature review. Int J Adolesc Med Health 2017;31 doi:10.1515/ijamh-2017-0037

6 Silva N, Pereira M, Otto C, et al. Do 8- to 18-year-old children/ adolescents with chronic physical health conditions have worse health-related quality of life than their healthy peers? A meta-analysis of studies using the KIDSCREEN questionnaires. Qual Life Res 2019;28:1725-50.

7 Hofmann SG, Gómez AF. Mindfulness-Based interventions for anxiety and depression. Psychiatr Clin North Am 2017;40:739-49.

8 Blanck P, Perleth S, Heidenreich T, et al. Effects of mindfulness exercises as stand-alone intervention on symptoms of anxiety and depression: systematic review and meta-analysis. Behav Res Ther 2018;102:25-35.

9 Greeson JM, Zarrin H, Smoski MJ, et al. Mindfulness meditation targets Transdiagnostic symptoms implicated in stress-related disorders: understanding relationships between changes in mindfulness, sleep quality, and physical symptoms. Evid Based Complement Alternat Med 2018;2018:1-10.

10 Xunlin NG, Lau Y, Klainin-Yobas P. The effectiveness of mindfulnessbased interventions among cancer patients and survivors: a systematic review and meta-analysis. Support Care Cancer 2020;28:1563-78.

11 Greeson JM, Chin GR. Mindfulness and physical disease: a concise review. Curr Opin Psychol 2019;28:204-10.

12 Lin J, Chadi N, Shrier L. Mindfulness-based interventions for adolescent health. Curr Opin Pediatr 2019;31:469-75.

13 Kabat-Zinn J. Wherever you go there you are: mindfulness meditation in everyday life. New York: Hyperion, 1994.

14 Kabat-Zinn J. Full catastrophe living: how to cope with stress, pain and illness using mindfulness meditation. New York: Delacorte, 1990.

15 Segal ZV, Williams JMG. Mindfulness-based cognitive therapy for depression: a new approach to preventing relapse. New York: Guildford Press, 2002.

16 Ahola Kohut S, Stinson J, Davies-Chalmers C, et al. Mindfulnessbased interventions in clinical samples of adolescents with chronic illness: a systematic review. J Altern Complement Med 2017;23:581-9.

17 Abujaradeh H, Safadi R, Sereika SM, et al. Mindfulness-based interventions among adolescents with chronic diseases in clinical settings: a systematic review. J Pediatr Health Care 2018;32:455-72.

18 Van der Gucht K, Takano K, Labarque V, et al. Mindfulnessbased intervention for adolescents and young adults after cancer treatment: effects on quality of life, emotional distress, and cognitive vulnerability. J Adolesc Young Adult Oncol 2017;6:307-17.

19 Chadi N, Kaufman M, Weisbaum E, et al. Comparison of an inperson vs. Ehealth mindfulness meditation-based intervention for 
adolescents with chronic medical conditions: a mixed methods study. J Adolescent Health 2018;62:S12.

20 Kratochwill TR, Levin JR. Enhancing the scientific credibility of single-case intervention research: randomization to the rescue. Psychol Methods 2010;15:124-44.

21 Rhoda DA, Murray DM, Andridge RR, et al. Studies with staggered starts: multiple baseline designs and group-randomized trials. Am J Public Health 2011;101:2164-9.

22 Hofmann SG, Hayes SC. The future of intervention science: ProcessBased therapy. Clin Psychol Sci 2019;7:37-50.

23 Csikszentmihalyi M. Flow and the foundations of positive psychology: the collected works of Mihaly Csikszentmihalyi. Dordrecht: Springer Netherlands, 2014.

24 Quené $\mathrm{H}$, van den Bergh $\mathrm{H}$. On multi-level modeling of data from repeated measures designs: a tutorial. Speech Commun 2004;43:103-21.

25 Crane RS, Brewer J, Feldman C, et al. What defines mindfulnessbased programs? The warp and the weft. Psychol Med 2017;47:990-9.

26 der Gucht KV, Takano K, Kuppens P, et al. Potential moderators of the effects of a school-based mindfulness program on symptoms of depression in adolescents. Mindfulness 2017;8:797-806.

27 Varni JW, Seid M, Kurtin PS. PedsQL 4.0: reliability and validity of the pediatric quality of life inventory version 4.0 generic core scales in healthy and patient populations. Med Care 2001;39:800-12.

28 Engelen V, Haentjens MM, Detmar SB, et al. Health related quality of life of Dutch children: psychometric properties of the PedsQL in the Netherlands. BMC Pediatr 2009;9:68.

29 de BE, van Dyck R, Marquenie LA. De DASS: Een vragenlijst voor het meten van depressie, angst en stress. [The DASS: A questionnaire for the measurement of depression, anxiety, and stress. Gedragstherapie 2001;34:35-53.

30 Lovibond SH, Lovibond PF. Manual for the depression anxiety stress scales. 2nd edn. Sydney: Psychology Foundation, 1995.

31 Willemsen J, Markey S, Declercq F, et al. Negative emotionality in a large community sample of adolescents: the factor structure and measurement invariance of the short version of the depression anxiety stress scales (DASS-21). Stress and Health 2011;27:e120-8.

32 Bastin M, Nelis S, Raes F, et al. Party pooper or life of the Party: dampening and enhancing of positive affect in a peer context. J Abnorm Child Psychol 2018;46:399-414.
33 Feldman GC, Joormann J, Johnson SL. Responses to positive affect: a self-report measure of rumination and dampening. Cognit Ther Res 2008;32:507-25.

34 Raes F, Daems K, Feldman GC, et al. A psychometric evaluation of the Dutch version of the responses to positive affect questionnaire. Psychol Belg 2013;49:293.

35 Ehring $\mathrm{T}$, Zetsche U, Weidacker $\mathrm{K}$, et al. The perseverative thinking questionnaire (PTQ): validation of a content-independent measure of repetitive negative thinking. J Behav Ther Exp Psychiatry 2011;42:225-32.

36 Ehring T, Raes F, Weidacker K, et al. Validation of the Dutch version of the perseverative thinking questionnaire (PTQ-NL). Eur J Psychol Assess 2012;28:102-8.

37 Raes F, Pommier E, Neff KD, et al. Construction and factorial validation of a short form of the Self-Compassion scale. Clin Psycho Psychother 2011;18:250-5.

38 Johnson C, Burke C, Brinkman S, et al. Development and validation of a multifactor mindfulness scale in youth: the comprehensive inventory of mindfulness Experiences-Adolescents (CHIME-A). Psychol Assess 2017;29:264-81.

39 De Vlieger P, Van den Bussche E, Eccleston C, et al. Finding a solution to the problem of pain: conceptual formulation and the development of the pain solutions questionnaire (PaSol). Pain 2006;123:285-93.

40 Jonsson U, Alaie I, Parling T, et al. Reporting of harms in randomized controlled trials of psychological interventions for mental and behavioral disorders: a review of current practice. Contemp Clin Trials 2014;38:1-8.

41 Hawkins NG, Sanson-Fisher RW, Shakeshaft A, et al. The multiple baseline design for evaluating population-based research. Am J Prev Med 2007;33:162-8.

42 Prinz JN, Bar-Kalifa E, Rafaeli E, et al. Imagery-based treatment for test anxiety: a multiple-baseline open trial. J Affect Disord 2019:244:187-95.

43 Van der Gucht K, Dejonckheere E, Erbas Y, et al. An experience sampling study examining the potential impact of a mindfulnessbased intervention on emotion differentiation. Emotion 2019;19:123-31.

44 Benjamini Y, Hochberg Y. On the adaptive control of the false discovery rate in multiple testing with independent statistics. $J$ Educ Behav Stat 2000;25:60-83. 